\title{
Dificuldades conceituais e matemáticas apresentadas por alunos de física dos períodos finais
}

\author{
Conceptual and mathematical difficulties presented by Physics students at the final semesters \\ of the course
}

\author{
Tiago Destéffani Admiral*
}

Instituto Federal de Educação, Ciência e Tecnologia Fluminense, Campos dos Goytacazes, RJ Brasil

Recebido em 2 de setembro de 2015. Aceito em 30 de setembro de 2015

\begin{abstract}
Este artigo descreve uma pesquisa realizada com estudantes de licenciatura em física de duas instituições públicas distintas. O grupo de 23 estudantes observados foi exclusivamente composto de alunos de sexto, sétimo e oitavo períodos. A pesquisa buscou identificar possíveis problemas conceituais dos alunos, suas habilidades para identificar o grau de dificuldade do problema e também averiguou secundariamente até que ponto os licenciandos dominam o cálculo como ferramenta para solução de problemas. Foi apresentado aos alunos um problema retirado de uma prova de vestibular de uma universidade federal. Essa questão, devido a um erro na elaboração, não tem como ser resolvida apenas com ferramentas matemáticas de Ensino Médio. Os alunos ainda responderam a um questionário semiestruturado que refletia suas percepções sobre o problema. Com base na análise de conteúdo do material escrito, obtido por meio da coleta de dados, percebemos que apenas dois estudantes obtiveram êxito na tarefa que lhes foi concedida, sendo que mais da metade deles falharam ao interpretarem conceitualmente o problema.
\end{abstract}

Palavras-chave: ensino de física, ensino de matemática, problemas conceituais.

This article describes a research with physics undergraduate students in two distinct public institutions. The group of 23 observed students was composed exclusively of students from sixth, seventh and eighth period. The research sought to identify possible conceptual problems of the students, their abilities to identify the level of difficulty of the problem; also, it was examined the extent that undergraduates dominate mathematical calculus as a tool for troubleshooting. It was presented to the students a problem taken from a federal university college entrance test. Due to an error in the preparation, this problem can only be solved with higher education mathematical tools. Students also answered a semi-structured questionnaire that identified their perceptions about the problem. Based on the analysis of the written collected material, we see that only two students were successful in the task, and more than half of them failed to interpret the problem conceptually.

Keywords: Physics education, Mathematics education, conceptual problems.

\section{Introdução}

A resolução de um problema de física pode envolver a mobilização simultânea de conceitos científicos, leis, relações entre grandezas distintas e ferramentas matemáticas. Essa combinação de habilidades demanda uma integração de conhecimentos diferentes, tarefa que, por vezes, pode ser a responsável pelo insucesso na tentativa de o aluno resolver um problema.

*Endereço de correspondência: tdesteffani@gmail.com.
As ferramentas matemáticas utilizadas na resolução são os elementos que nos permitem classificar uma questão de física como adequada para avaliar um aluno de Ensino Médio ou superior. Em linhas gerais, grande parte dos conceitos de física apresentados aos alunos de Ensino Médio são os mesmos utilizados por alunos de graduação na resolução de problemas de física básica. A principal diferença está no fato de que aos alunos de ensino superior são apresentados casos mais próximos de situações reais, e definições matematicamente mais precisas. 
Entretanto, de acordo com Barbeta e Yamamoto [1], as dificuldades enfrentadas pelos alunos no estudo de física são, muitas vezes, erroneamente atribuídas à deficiência dos alunos em manipular o ferramental matemático. Os autores aplicaram um teste adaptado de outro, conhecido como Mechanics Baseline Test, e identificaram que grande parte dos alunos do grupo estudado demonstrou conhecimentos de senso comum em relação a alguns conceitos físicos.

Para alguns autores $[1,2]$, os problemas de ordem conceitual, apresentados pelos alunos no ensino superior, são determinantes para o insucesso na tentativa de resolver problemas. Além disso, os autores defendem que a física e a matemática estão profundamente relacionadas, e que os conhecimentos matemáticos têm influência na compreensão dos conceitos físicos. Uma vez que grande parte dos conceitos físicos é baseada em modelos matemáticos, o diálogo entre as duas áreas tende a produzir efeitos positivos no ensino de física. É essencial para um aluno do curso de licenciatura em física, na qualidade de futuro professor, identificar as condições e suposições conceituais que estão implícitas na situaçãoproblema de uma questão e, com base nessa análise, selecionar as ferramentas necessárias à solução do problema.

Mesmo que um aluno tenha determinado corretamente a forma de resolver um problema, ele ainda precisa dominar as ferramentas necessárias para resolvê-lo. Na falta de um dos requisitos, o problema não será resolvido. De acordo com Pietrocola [2], a relação entre o conhecimento matemático e físico vai muito além de uma mera dependência mecânica, conhecimento matemático possui também o papel de aperfeiçoar a compreensão de modelos e conceitos físicos de forma dialética.

Esta pesquisa buscou determinar até que ponto o grupo de licenciandos observados foi capaz de identificar a real complexidade de um problema apresentado mediante a interpretação física adequada à situação. Como objetivo complementar, a pesquisa mostrou que a maioria dos alunos não foi capaz de identificar corretamente as ferramentas matemáticas necessárias para a sua solução e verificou se a manipulação do ferramental matemático foi bem-sucedida.

\section{Análise da questão proposta}

Foi proposta aos alunos uma questão de física, cuja origem não foi identificada. A pesquisa analisou inclusive a opinião dos alunos em relação à classificação da questão de Ensino Médio ou superior. A questão fez parte de um vestibular de uma universidade federal.

O objetivo do artigo não é tecer críticas à instituição e/ou entidade elaboradora de itens, por isso não será mencionada aqui a origem da questão. A pressão para que a elaboração de uma questão de vestibular seja inédita, desafiadora, coerente, realística e contextualizada para nível médio não é uma exigência nada trivial e, por vezes, inviável, dependendo do conteúdo abordado, dadas principalmente as limitações das ferramentas matemáticas apresentadas pelos alunos.

Mais adiante é apresentada a questão tal como foi utilizada originalmente como questão de vestibular e, em seguida, a solução proposta por nós, de acordo com a situação descrita.

1 - Observe esta figura:

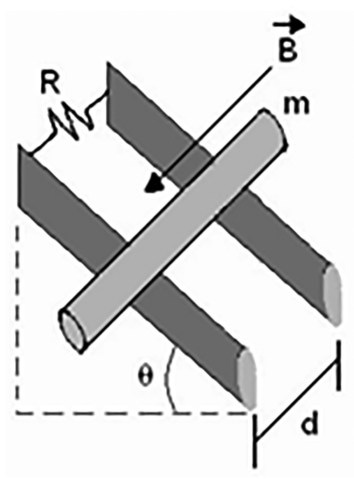

Nessa figura, duas barras metálicas fixas e separadas por uma distância $d$ igual a $20 \mathrm{~cm}$ formam um plano, cuja inclinação em relação ao plano horizontal é igual $\theta=30^{\circ}$. No espaço, tem-se um campo magnético uniforme $(\mathbf{B})$ igual a 1,5 $T$, perpendicular ao plano inclinado e entrando nele, conforme demonstrado na figura. As duas barras são ligadas por um resistor com resistência $R$ igual a $2 \Omega$. Outra barra metálica, de massa $m=100 \mathrm{~g}$, desloca-se sobre as barras fixas, a partir do repouso e dos extremos superiores das barras inclinadas e também sem atrito. Suponha que as resistências das barras sejam desprezíveis e a aceleração da gravidade no local seja igual a $10 \mathrm{~m} / \mathrm{s}^{2}$. Assinale a alternativa que contém o valor correto do instante em que a barra em movimento atinge a velocidade terminal.
A) 5,00 segundos.
B) 1,00 segundo.
C) 4,00 segundos. 
D) 2,22 segundos.

Solução: inicialmente a barra se encontra em repouso em relação ao solo e, abandonada a partir dessa condição, é acelerada sob a influência da gravidade. Considerando a perspectiva de um observador que está posicionado na parte superior do plano formado pelos trilhos, e, paralelamente às linhas de campo, podemos representar as forças que atuam sobre a barra pela Fig. 1 .

Conforme a barra desliza sobre os trilhos, o fluxo magnético, no circuito formado pelos trilhos, barra e resistor, aumentará gradativamente. Também aumentará a força magnética que atua sobre a barra que, por sua vez, é diretamente proporcional à corrente induzida no circuito. Exatamente o fato de a força magnética não ser constante faz com que essa questão não seja resolvida por um aluno de Ensino Médio. De acordo com a segunda Lei de Newton, temos que, na direção $\hat{x}$,

$$
\begin{array}{r}
m \cdot g \cdot \operatorname{sen} \theta(+\hat{i})+B \cdot d \cdot I(t)(-\hat{i})= \\
m \cdot a(t)(+\hat{i}),
\end{array}
$$

em que $m$ é a massa da barra, $g$ é a aceleração da gravidade, $\theta$ é o ângulo de inclinação do plano, $d$ é a distância entre as barras laterais que formam o plano e $B$ é o módulo do campo magnético. Cada termo está multiplicado por um vetor unitário que indica seu respectivo sentido, visto que todos compartilham a direção. Vale ressaltar que a corrente

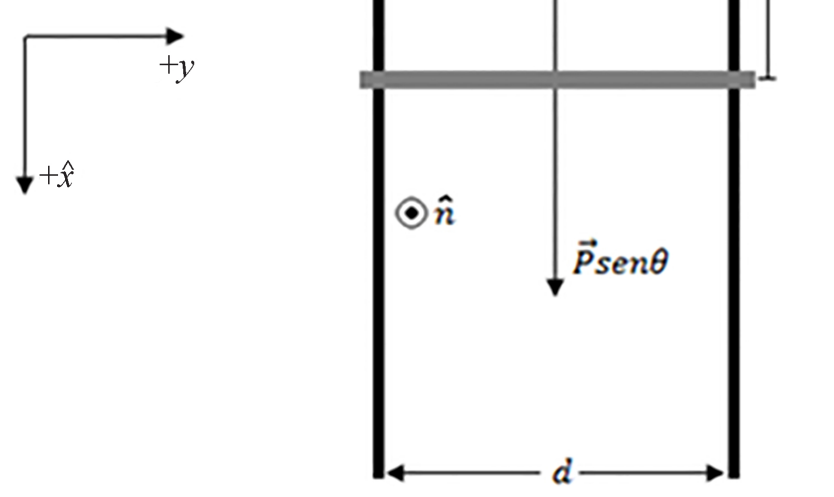

Figura 1: Esquema do plano inclinado que contém os triIhos e a barra, visão superior. elétrica citada na equação acima varia com o tempo. Segundo a Lei de Faraday, temos que

$$
\varepsilon=-\frac{d \Phi}{d t}
$$

O módulo do fluxo magnético $(\Phi)$, no caso particular em que o campo magnético é uniforme, pode ser "obtido pelo produto entre o módulo do campo magnético $\mathbf{B}$, da área $A$, e o cosseno do ângulo $\theta$ formado entre os vetores $\hat{n}$ e $\mathbf{B}$ " [3]. Desse modo

$$
\Phi=B \cdot A(t) \cdot \cos (\theta) .
$$

$\mathrm{O}$ ângulo entre os vetores $\hat{n}$ e $\mathbf{B}$ pode ser obtido através da Fig. 1. Substituindo a Eq. (3) na Eq. (2) obteremos

$$
\varepsilon=-\frac{d[B \cdot A(t) \cdot \cos (\pi)]}{d t}
$$

Simplificando a Eq. (4) teremos

$$
\varepsilon=B \cdot d \cdot \frac{d[x(t)]}{d t} .
$$

O termo $\left[\frac{d[x(t)]}{d t}\right]$, que representa a taxa variação da dimensão $x$ em relação ao tempo, define a velocidade instantânea da barra $[v(t)]$ durante o movimento. Utilizando primeira lei de $\mathrm{Ohm}$, temos que a corrente induzida é dada por

$$
I(t)=\frac{B \cdot d}{R} v(t)
$$

em que $I(t)$ é a corrente induzida e $R$ é a resistência elétrica fornecida como parâmetro no problema. Ao substituirmos a Eq. (6) na Eq. (1), e reorganizarmos, teremos que, em módulo,

$$
\frac{d[v(t)]}{d t}+\left(\frac{B^{2} d^{2}}{R \cdot m}\right) \cdot v(t)-g \cdot \operatorname{sen} \theta=0 .
$$

Para simplificarmos a solução desta EDO, usaremos

$$
p=\left(\frac{B^{2} d^{2}}{R \cdot m}\right), \text { e } q=g \cdot \operatorname{sen} \theta .
$$

Pelo método de solução do fator integrante, de acordo com William e Richard [4], temos que a solução geral dessa equação diferencial ordinária de coeficientes constantes é da forma

$$
v(t)=e^{-\int^{p} \cdot d t} \cdot\left(\int^{q} \cdot e^{\int^{p}} \cdot d t \cdot d t+C\right) .
$$


Ao resolvermos a expressão (9), obteremos como solução

$$
v(t)=\frac{q}{p}+C \cdot e^{-p \cdot t} .
$$

Aplicando a condição de contorno $v(0)=0$ para determinar a constante de integração, resultará em

$$
v(t)=\frac{q}{p} \cdot\left(1-e^{-p \cdot t}\right) .
$$

Ao substituirmos na Eq. (11) as constantes $p$ e $q$, definidas na Eq. (8), obteremos a equação

$$
v(t)=\frac{R \cdot m \cdot g \cdot \operatorname{sen} \theta}{B^{2} d^{2}} \cdot\left[1-e^{-\left(\frac{B^{2} d^{2}}{R \cdot m}\right) t}\right](+\hat{i}) .
$$

A Eq. (12) representa a forma como a velocidade da barra varia com o tempo. A determinação da velocidade limite é obtida analisando o comportamento dessa função para valores de tempo muito grandes, nesse caso teremos, em módulo,

$$
\lim _{t \rightarrow \infty} v(t)=\frac{R \cdot m \cdot g \cdot \operatorname{sen} \theta}{B^{2} d^{2}} .
$$

Ao substituirmos os valores numéricos oferecidos pelo enunciado da questão, na expressão (13), constatamos que a velocidade limite é de $11,11 \mathrm{~m} / \mathrm{s}$. Observe que, decorrido o valor de tempo sugerido pelo gabarito da prova (letra d, 2,22 s), a velocidade da barra terá atingido $63,2 \%$ do valor da velocidade limite. É relevante deixar aqui registradas duas observações sobre os itens: em primeiro lugar (e menos importante), os itens não apresentam ordenamento crescente, conforme recomendam as orientações para elaboração dos itens na resposta; em segundo lugar, os itens são todos valores inteiros, exceto o item $\mathrm{D}$, o que, de certa forma, denuncia que o item tem grande chances de ser a resposta 'correta'.

Embora seja bem diferente do valor real da velocidade limite, esse valor sugerido pelo gabarito, não coincidentemente, também possui um significado físico. Para sistemas que decaem em regime exponencial, como a descarga de um capacitor, o tempo que decorre, até que o capacitor tenha descarregado $63,2 \%$, é conhecido como tempo característico ou constante de tempo. O tempo característico, usualmente representado pela letra grega tau $(\tau)$, corresponde ao tempo em que o sistema começa a responder mais acentuadamente ao regime de equilíbrio.
Analogamente, a resolução de um problema de circuito RC apresenta uma EDO da seguinte forma

$$
\frac{d q}{d t}+\frac{1}{R C} q=\frac{V_{e}}{R},
$$

onde que $q$ é função do tempo e os parâmetros $R, C$ e $V_{e}$ são constantes. Ao solucionarmos essa EDO, com condições de contorno similares ao do nosso problema, obteremos como solução

$$
q=C V_{e}\left(1-e^{-\frac{t}{R C}}\right) .
$$

Da mesma forma que nessa equação, em que o termo RC é chamado de tempo característico, percebemos que, em nossa solução, analogamente a constante $(p)$, que acompanha o termo $v(t)$ na EDO, também aparece na resposta final, não coincidentemente ela fornece o valor $(2,22 \mathrm{~s})$ da resposta sugerida pelo gabarito. Isso decorre da aproximação sugerida implicitamente pelo elaborador da questão, para solucionar o problema sem utilizar cálculo diferencial, ao assumir que o módulo da aceleração é constante $(g \cdot \operatorname{sen} \theta)$.

Entretanto, essa aproximação é imprecisa tanto sob o aspecto numérico quanto conceitual. Esse problema apresenta uma aceleração cujo módulo varia com o tempo. Tal variação é obtida por meio da derivada da Eq. (12) em função de $t$

$$
a(t)=g \cdot \operatorname{sen} \theta \cdot e^{-\left(\frac{B^{2} d^{2}}{R \cdot m}\right) t} .
$$

Para valores de tempo muito pequenos, a aceleração se aproxima muito do valor sugerido para a aproximação; entretanto, quanto maior o tempo decorrido, tanto mais próxima de zero será a aceleração, o que nos leva a deduzir que, quanto maiores forem os valores de tempo, mais próximo o sistema estará de executar um movimento uniforme. A explicação dessa afirmação pode ser obtida quando investigamos o limite da função $a(t), \operatorname{com} t$ tendendo ao infinito

$$
\lim _{t \rightarrow \infty} a(t)=0 .
$$

A consequência imediata desse resultado é que, quanto maiores os valores de tempo, tanto mais próxima de zero será a aceleração do sistema, o que configura o movimento como uniforme. A Fig. 2 ilustra como varia o módulo da aceleração com o tempo. 


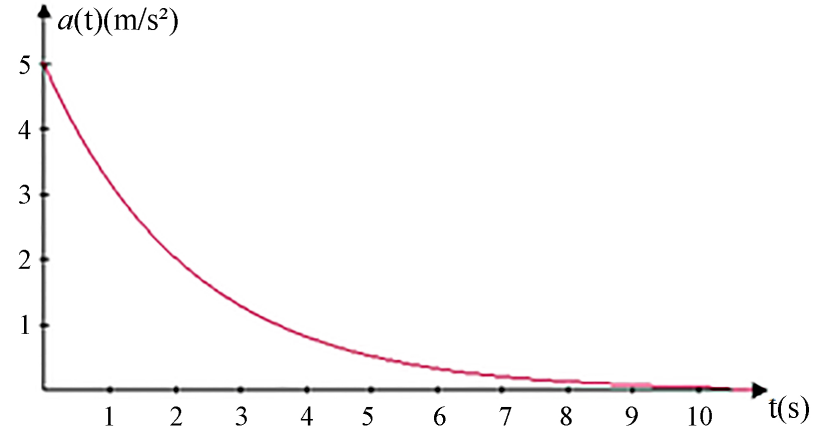

Figura 2: Aceleração vs. tempo.

\section{Metodologia e coleta de dados}

A pesquisa foi realizada com um total 23 alunos do curso de licenciatura em física de duas instituições distintas: Instituto Federal Fluminense, campus Centro, Campos dos Goytacazes - RJ, e Universidade Estadual do Norte Fluminense, Campos dos Goytacazes - RJ.

Os estudantes selecionados para participarem da pesquisa foram os alunos que estavam cursando o sexto, sétimo e oitavo períodos. Essa condição se justifica, pois, para que o estudante desenvolva corretamente a questão, ele deve ter cursado as disciplinas de cálculo e as disciplinas básicas de mecânica e eletromagnetismo.

Embora o número total de estudantes observados seja relativamente pequeno, essas duas instituições atendem uma grande região, que engloba o sul do estado do Espírito Santo e norte fluminense. Nessas regiões, essas são as principais instituições públicas que oferecem o curso de licenciatura em física. Por isso, a representatividade dos dados se reforça em virtude da dimensão geográfica que essas instituições atendem. Somando-se a esse fato, é notória a informação de que usualmente se encontram poucos alunos [5] nos períodos finais dos cursos de física. A coleta de dados foi realizada no período de $3 / 2015$ a $6 / 2015$.

Para a coleta de dados, foi utilizado um questionário semiestruturado. Minayo [6] define que um questionário semiestruturado "combina perguntas fechadas (ou estruturadas) e abertas (discursivas), em que o entrevistado tem a possibilidade de discorrer o tema proposto, sem respostas ou condições prefixadas pelo pesquisador". Esse formulário contém o problema e cinco questões sobre o problema e suas características, sendo quatro questões objetivas e uma discursiva. Os alunos foram orientados a responder ao questionário com tranqüilidade e lhes foi esclarecido que não se tratava de uma avaliação ou teste, mas de uma pesquisa de opinião, e ainda que eles se sentissem à vontade para tentar resolver a questão da forma como acreditavam ser a correta. Os alunos tiveram um tempo de aproximadamente 40 minutos para resolver o problema e responder ao questionário.

\section{Resultados e discussão}

Como não faz parte dos objetivos deste trabalho realizar nenhum tipo de comparação referente ao desempenho de alunos de instituições diferentes, os dados foram agrupados e tratados como único conjunto. Todos já haviam cursado as disciplinas necessárias para conseguir resolver o problema, foram apresentados ao mesmo problema e tiveram o mesmo tempo para resolvê-lo. Desse modo, acreditamos que seja razoável agrupar os dados e fazer a análise em conjunto. A Tabela 1 mostra a distribuição das respostas dos alunos.

Ao analisarmos as respostas dos alunos aos itens, constatamos que oito assinalaram, na primeira questão do questionário, que o problema era adequado a um aluno do Ensino Médio. Esse grupo de alunos que representam um terço do total obviamente não percebeu as implicações mais complexas do problema. Os demais 15 alunos responderam que esse problema é mais adequado a um aluno de ensino superior. Mais adiante, ao analisarmos a questão discursiva, poderemos inferir mais precisamente o que levou os alunos a responder aos itens dessa maneira.

Quando perguntados sobre a possibilidade de o problema ter apresentado dados suficientes para a resolução, 18 alunos responderam que sim e apenas cinco responderam que não. Todos os cinco alunos que responderam que a questão não apresentou dados suficientes para resolução também assinala-

Tabela 1: Número de alunos que responderam a cada item das questões.

\begin{tabular}{lccccc}
\hline Questões/respostas aos itens & 1 & 2 & 3 & 4 & 5 \\
Questão 1 & 8 & 15 & - & - & - \\
Questão 2 & 18 & 5 & - & - & - \\
Questão 3(a) & 0 & 1 & 3 & 11 & 8 \\
Questão 3(b) & 3 & 3 & 7 & 8 & 2 \\
Questão 4 & 7 & 5 & 11 & - & - \\
\hline
\end{tabular}


ram, no item anterior, que o problema era adequado ao Ensino Médio. Os 18 alunos restantes que assinalaram que o problema ofereceu todos os dados necessários à solução pareciam ter compreendido a situação-problema corretamente. Entretanto, com a análise dos outros itens do questionário, fica evidente que apenas uma pequena parte soube justificar corretamente essa escolha

A terceira pergunta do questionário possuía dois itens: o primeiro perguntava sobre o nível de dificuldade desse problema do ponto de vista de um aluno de Ensino Médio; o segundo pergunta a mesma coisa, mas para um aluno de ensino superior. Na escala de 1 a 5 , não houve marcação na opção 1 , que correspondia a "muito fácil". Houve uma marcação na opção 2, que correspondia a "fácil", entretanto o aluno que assinalou essa alternativa havia marcado anteriormente que a questão tratava de uma questão de Ensino Médio e também que havia dados suficientes para resolvê-la. Três alunos consideraram que essa seria uma questão de nível de dificuldade razoável para um aluno de Ensino Médio, enquanto 11 alunos afirmaram que essa questão seria difícil para um aluno de Ensino Médio.

Percebemos que, até aqui, independentemente do nível de dificuldade atribuído à questão, esses 15 alunos acreditam que um aluno de Ensino Médio pode resolver esse problema de maneira correta. Apenas oito alunos assinalaram o item cinco, que correspondia a "impossível", para um aluno de Ensino Médio.

A quarta questão perguntava se o licenciando, na condição de futuro professor, usaria o problema em suas avaliações para Ensino Médio. Sete alunos assinalaram que usariam a questão na íntegra, cinco afirmaram que não usariam a questão e 11 alunos indicaram que usariam com adaptações.

Podemos categorizar as informações contidas na questão 5 de acordo com seus núcleos de significados [7], e essa categorização pode ser organizada conforme critérios previamente estabelecidos, semelhanças ou analogias entre as falas [8]. Para que se mantivesse o sigilo, os alunos foram identificados com números de 1 a 23. Essa numeração não foi arbitrária, está em ordem crescente de acordo com o desempenho. A classificação obedeceu aos critérios de desempenho do aluno na resolução da questão, a saber:
- Identificou corretamente a natureza das forças que atuam no sistema (aspecto conceitual);

- Definiu corretamente o somatório de forças, identificando a força magnética e aceleração como funções do tempo;

- Identificou corretamente quais ferramentas matemáticas seriam necessárias para a solução;

- Executou com êxito as operações matemáticas necessárias.

Quando os alunos apresentaram desempenhos semelhantes, conseguindo atingir as mesmas habilidades, utilizamos como critério de desempate, a "coerência" nas respostas do questionário. Esse critério passou pela comparação entre as respostas dadas no questionário e o desempenho na resolução da questão, por exemplo, o Aluno 1, que afirmou que a questão era de nível médio (pela resposta da questão 1); que tinha dados suficientes (pela questão 2); classificou a questão como fácil para um aluno de superior (questão $3-$ b), mas não conseguiu desenvolver nenhum dos critérios de desempenho de maneira correta.

Dos 11 alunos que afirmaram que usariam o problema para avaliar seus alunos de Ensino Médio, 10 responderam à questão 5 . Do grupo de alunos que responderam à quinta questão, três sugeriram adaptações irrelevantes, sob o aspecto da adequação conceitual e matemática do problema para o aluno de Ensino Médio, como a qualidade da figura, por exemplo.

Mesmo que o aspecto visual do problema apresente algum tipo de imprecisão, não é a qualidade da figura a responsável pelo fato de um aluno de Ensino Médio não poder resolver o problema. Organizando as demais sugestões, encontramos dois tipos de padrões diferentes nas respostas. Uma categoria de sugestões consiste basicamente no grupo de alunos que alegaram que a questão deveria apresentar uma "contextualização" ou uma "situação problema" mais elaborada. Pudemos observar similaridades que convergem nesse sentido em cinco respostas e, para ilustrarmos, podemos indicar a resposta do Aluno 10:

Aluno 10: "Para ficar melhor elaborada a questão deveria conter uma aplicação do cotidiano do aluno, uma situação prática para contextualização" 
Por fim um grupo, constituído de apenas dois alunos, fez sugestões que indicam que eles realmente perceberam o motivo pelo qual a questão é impossível de ser resolvida por um aluno de Ensino Médio. As respostas dos alunos foram agrupadas nessa categorização por conterem a explicação detalhada da força magnética que age sobre a barra, ou indícios de que os alunos perceberam que ela varia com o tempo. As respostas estão transcritas abaixo:

Aluno 22: "Mudaria a situação para uma força constante puxando na horizontal, pois a força magnética não é uniforme até o equilíbrio".

Aluno 23: "Não pediria o tempo até que entre em MRU porque o aluno não saberia calcular com uma aceleração variável, cobraria só o valor da velocidade limite".

Embora não esteja escrito explicitamente na resposta do Aluno 23, ele interpretou corretamente que a força magnética que atua sobre a barra não é constante até que ela atinja o movimento uniforme. Podemos garantir isso pelo fato de que esse foi o único aluno que conseguiu resolver com sucesso a questão da forma como foi apresentada neste trabalho. Outros alunos conseguiram determinar a forma de resolvê-la, mas não obtiveram êxito na manipulação matemática.

Identificamos que o principal problema de interpretação conceitual apresentado pelos alunos foi a não compreensão das circunstâncias que tornam a força magnética que atua sobre a barra variável. Com base na análise das respostas dos alunos, erroneamente a força foi considerada constante.

Por fim, apenas dois alunos, conseguiram determinar o caminho exato para a solução do problema e as ferramentas matemáticas necessárias e utilizálas corretamente. $\mathrm{Na}$ realidade, o resultado final foi obtido apenas pelo Aluno 23, e o Aluno 22 não conseguiu determinar corretamente a constante de integração do P.V.I. após a solução da E.D.O. Mas podemos considerar que ele, apesar do deslize, tem o conhecimento que é necessário para analisar corretamente e resolver o problema.

\section{Considerações finais}

No intuito de contribuir com resultados citados anteriormente por pesquisas realizadas em outras regiões e realidades, esta pesquisa sugere que as dificuldades na formação inicial do aluno de física, no que diz respeito às dificuldades conceituais, não são pontuais.

Em face dos dados obtidos da observação, pudemos perceber um grande percentual de alunos com dificuldades em aferir conceitualmente o grau de dificuldade da questão apresentada, bem como executar o cálculo necessário para resolvê-la. Menos da metade dos estudantes demonstrou o conhecimento esperado para o nível em que se encontram.

Fazendo uma reflexão sobre o contexto da pesquisa, devemos estar cientes de que diversos elementos são passíveis de análise e influenciam no desempenho dos alunos. Os aspectos qualitativos, referentes à observação durante a aplicação do questionário, sugerem fortemente que os alunos se empenharam na tarefa que lhes foi concedida. Ao analisar esse comportamento, estamos convencidos de que as respostas dos alunos refletem, de maneira muito próxima da realidade, o nível de conhecimento no momento da aplicação do questionário.

$\mathrm{O}$ isolamento disciplinar entre física e Matemática certamente apresenta indicativos de ineficiência que também foram relatados em trabalhos anteriores já citados neste artigo. É possível que a excessiva mecanização no ensino de cálculo, que pode apresentar certa eficiência no aspecto procedimental em relação aos algoritmos, dificulte ao aluno estabelecer as relações de aplicabilidade no campo da física, as quais, muitas vezes, subsidiam o próprio conhecimento teórico.

Esforços bem-sucedidos estão sendo apresentados, cada vez mais frequentemente, no intuito de sanar essa dificuldade dos alunos. Pesquisas recentes [9] sugerem que cursos de cálculo direcionado a alunos de cursos específicos tendem a apresentar resultados positivos de aprendizagem. Ignorar a dificuldade do aluno atribuindo a culpa à sua formação anterior é, muito provavelmente, uma atitude que de nada servirá para fazer que o professor conclua com êxito seu trabalho, que é ensinar.

\section{Referências}

[1] V.B. Barbeta e I. Yamamoto, Revista Brasileira de Ensino de Física 24, 324 (2002).

[2] M. Pietrocola, Caderno Catarinense de Ensino de Física 19, 88 (2002). 
[3] P.A. Tipler, Física para Cientistas e Engenheiros - Volume 2 - Eletricidade e Magnetismo (Editora LTC, Rio de Janeiro, 2009).

[4] William E. Boyce e Richard C. Diprima, Equações Diferenciais Elementares e Problemas de Valores de Contorno (LTC, São Paulo, 2006), $8^{\mathrm{a}}$ ed, p. 18.

[5] S.T. Gobara e J.R.B. Garcia, Revista Brasileira de Ensino de Física 29, 519 (2007).

[6] Maria Cecília de Souza Minayo (org), Pesquisa Social: Teoria, Método e Criatividade (Vozes, Rio de Janeiro, 1994), 20 ${ }^{\mathrm{a}}$ ed.

[7] L. Bardin, Análise de Conteúdo (Edições 70, Lisboa, 2004), $3^{\text {a }}$ ed.

[8] Roque Moraes, Revista Educação 22, 7 (1999).

[9] M.C.P. Santarosa e M.A. Moreira, Investigações em Ensino de Ciências 16, 317 (2011). 